IP Periodica Polytechnica Chemical Engineering

60(4), pp. 244-251, 2016

DOI: 10.3311/PPch.9101

Creative Commons Attribution (i)

RESEARCH ARTICLE

\section{Optical Simulations of Self-assembly Relevant Gold Aggregates: A Comparative Study}

\author{
Dániel Zámbó ${ }^{1}$, András Deák $^{1 *}$
}

Received 17 February 2016; accepted after revision 31 May 2016

\begin{abstract}
In this study, visible light extinction spectra of different gold nanoparticle assemblies were simulated using boundary element method (BEM) in order to investigate the optical properties of structures which determine the experimentally measured spectra during the self-assembly of the particles. Numerous different optically dominant particle arrangements can evolve depending on the underlying physicochemical mechanism of the clustering process itself: one-dimensional chains, two-dimensional arrays or three-dimensional clusters can be formed in the solutions or at interfaces. Experimentally the aggregation or clustering of gold nanoparticles can be conveniently followed by spectroscopic techniques due to the plasmon coupling related profound effect of particle aggregation on the visible extinction spectrum. However, the measured spectra usually contain the optical response of various aggregate structures. Additionally, small changes of the interparticle distance can have a significant impact on the frequency of the coupled mode. In order to assess the contribution of the different structures to the experimentally measurable extinction spectra during particle clustering, different model structures (chains, array and 3D-cluster) have been simulated, where the distance between the particles was varied as well.
\end{abstract}

\section{Keywords}

gold nanoparticles, optical simulations, boundary element method, nanoparticle chains, 2D heptamer, 3D cluster, self-assembly
${ }^{1}$ Institute for Technical Physics and Material Science,

Centre for Energy Research,

Hungarian Academy of Sciences

H-1525 Budapest, P.O.B. 49, Hungary

*Corresponding author, e-mail: deak.andras@energia.mta.hu

\section{Introduction}

Gold nanoparticles feature strong extinction in the visible wavelength range due to the evolution of localized surface plasmon resonance (LSPR) upon interaction with visible light, which can be exploited in the field of sensing [1-3], catalysis $[4,5]$, and biomedical applications $[6,7]$. Since the LSPR of gold nanoparticles manifests itself in a pronounced extinction peak in the visible, it can be conveniently investigated using ensemble spectroscopy techniques. The optical response of the particles on the other hand is tunable: the peak position of LSPR is sensitive to the size [8] and shape [9, 10] as well. Organizing the individual gold building blocks into self-assembled structures can open route towards new properties and applications [11]. In the self-assembly processes, 1D-chains (or small oligomers), 2D-arrays or 3D-compact nanoparticle clusters [12] are most commonly obtained. These nanostructures enable tuning of the optical response [13-15] for new type of applications, such as colorimetric assays [16] or based on SERS enhancement [17]. The clustering of the particles and the evolution of the different assemblies can be followed by UV-Vis spectroscopy. There are several literature examples, where assignment of the evolved nanoparticle cluster structure has been carried out solely based on spectroscopic characterization (or by naked eye due to the color change) and the identification of dimers, trimers [13], chains [18, 19] or larger clusters [15] based on the extinction spectra has been claimed. Two-dimensional nanoparticle arrays have been also reported with potentially useful optical properties due to the collective plasmonic oscillations [20, 21].

Besides the structure or size [22] of the particle cluster, the interparticle distance between the building blocks [21, 23] has also a profound effect on the evolving coupled mode. It has to be emphasized that in a real experiment - independently of the resulting structure - the clustering process usually generates a distribution of structures. Consequently, a multitude of coupled modes can exist simultaneously that can be excited by the external source. However, the overall optical response of the system is determined by the optically dominant centers [19], which is responsible e.g. for the presence of a 'distinct' coupled mode in the extinction spectrum during the aggregation of gold 
nanoparticles. Hence it is important to know, what the individual spectral response and their contribution to the overall optical signal of the different cluster types is.

From this point of view, simulation methods that enable the calculation of the optical response of such clusters are of great use, since they represent a convenient way to construct and calculate particle assemblies in silico. A wide range of method is used to simulate the electromagnetic properties of noble metal structures, relying on the solution of full Maxwell's equations. The most commonly used methods are the finite-difference time-domain (FDTD) [24], the finite element method (FEM) [25], the discrete dipole approximation (DDA) [26], and the boundary element method (BEM) [27]. While the first three approaches suffer from the drawback of volume discretization, BEM works only with surface elements, thus the computational time can be reduced significantly. If the surfaces are subdivided to sufficient number of elements, the calculations can have the same quality as for volume based methods.

In this paper we demonstrate BEM simulations of extinction spectra for linear chain, 2D array and 3D clusters made from gold nanoparticles (with diameters of 18 and $40 \mathrm{~nm}$ ) in the visible wavelength range. The results emphasize that spectroscopy alone is not adequate to conclude on the precise assembly structure, other imaging techniques should be used as well: similar optical response from essentially different nanostructures with different interparticle separations can be easily obtained in an experiment.

\section{Methods and simulation setup}

Optical simulation of gold nanoparticles and their assemblies was performed in Matlab® using the MNPBEM toolbox [28] based on boundary element method (BEM). MNPBEM was developed for the simulation of the electromagnetic properties of plasmonic nanoparticles, where the particles are embedded into an environment with homogeneous dielectric properties and are separated by well defined, abrupt interfaces and provides accurate results for the particle sizes concerned in the present work. During the calculations retardation effects have been also taken into account.

Three main geometries have been considered: linear chains, 2D monolayers and 3D clusters with internal order. For 1D chains, the number of constituent particles was varied from 2 to 13; for 2D arrays and 3D cluster the number of building blocks was 7 and 13, respectively. The interparticle distance was also varied from 0.2 to $3.8 \mathrm{~nm}$ and from 0.8 to $10 \mathrm{~nm}$ for $18 \mathrm{~nm}$ and $40 \mathrm{~nm}$ particles, respectively. These distance values were chosen based on our earlier work [12]. It is important to point out, however, that for the smaller sized particles for particle-particle separations below $0.5 \mathrm{~nm}$, the calculated spectra neglect nonlocal effects, and hence enable only qualitative conclusions on the associated spectral changes.
The nanoparticle surface was discretized using triangles, each nanoparticle surface was subdivided using 400 vertices. The embedding medium was set to water (dielectric constant: 1.776), while for the gold particles the dielectric function from Johnson and Christy were used [29]. Figure 1 shows the schematic representation of simulation setup with the discretized nanoparticle surface.

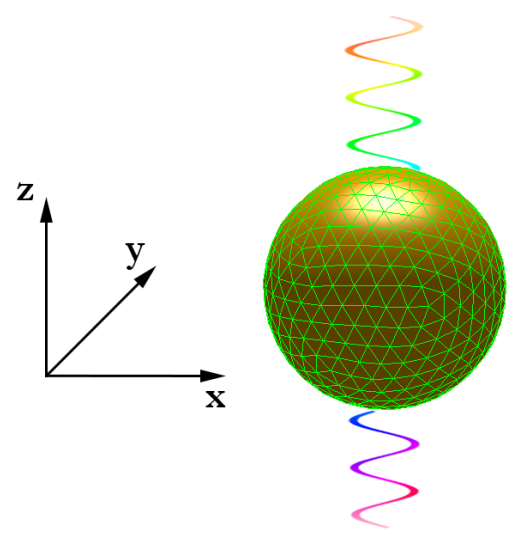

Fig. 1 Schematic representation of simulation concept: nanoparticle surface was subdivided using 400 vertices (green triangles represent the surface elements), the direction of irradiation was parallel to the $\mathrm{z}$ axis, and the light was $\mathrm{X}$-polarized for all geometries.

Figure 2 represents the model structures: linear chains were built from different numbers of building particles (2-13), 2D arrays contained 7 particles (characteristic building block of a hexagonal closed packed 2D structure), and the $3 \mathrm{D}$ clusters consisted of 13 nanoparticles in closed packed arrangement (A-B-A type structure).

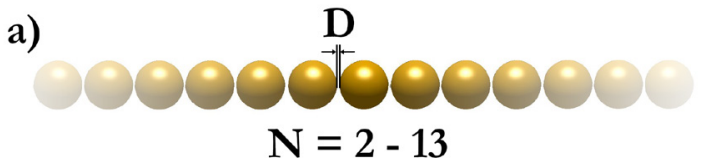

b)

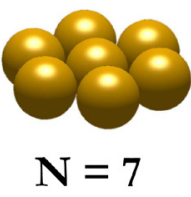

c)

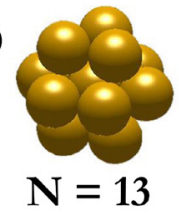

Fig. 2 Schematic representations of simulated model structures. $\mathrm{N}$ is the number of the gold nanoparticles in the structure, D is the interparticle separation distance.

For the geometrical arrangement of the 2D and 3D clusters, the coordinates for the particle centers as shown in Fig. 3 were used. 


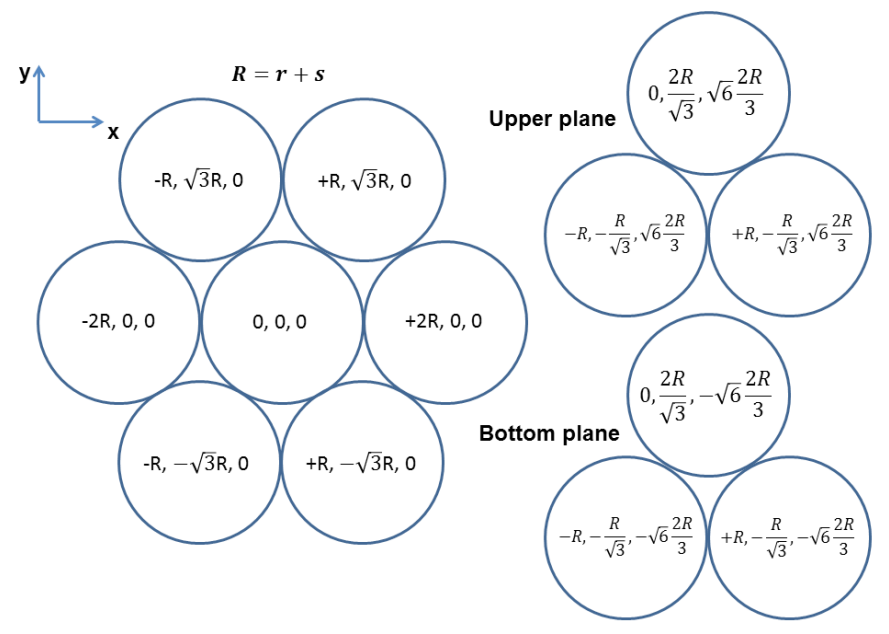

Fig. 3 Spatial coordinates of nanoparticles in an A-B-A type 3D cluster. Coordinates are $\mathrm{x}, \mathrm{y}$ and $\mathrm{z}$, respectively. $\mathrm{r}$ is the radius of particle, $\mathrm{s}$ is the half of the separation distance. The coordinates in the left panel have been used for the 2D heptamers.

As reported by us earlier [12], the 3D clustering of gold nanoparticles in aqueous medium can be carried out by modulating the effective range of steric repulsion between polyethylene glycol (PEG) grafted particles. Hence we restricted the interparticle separations between values derived from the thickness of PEG brush at the nanoparticle surface from the above mentioned reference. Table 1 summarizes the initial brush thicknesses and the applied separation distances (equaling to twice the brush thickness), as well as the reasonable minimal value for the separation as derived from interaction calculations. The detailed description of the procedure can be found in Ref. [12].

Table 1 Applied parameters in simulations: diameter of nanoparticles, polymer brush thicknesses for the two model systems and the extrema of separation distance values.

\begin{tabular}{llll}
\hline $\begin{array}{l}\text { NP diameter } \\
(\mathrm{nm})\end{array}$ & $\begin{array}{l}\text { Polymer brush } \\
\text { thickness }(\mathrm{nm})\end{array}$ & $\mathrm{D}_{\max }(\mathrm{nm})$ & $\mathrm{D}_{\min }(\mathrm{nm})$ \\
\hline 18 & 1.9 & 3.8 & 0.2 \\
40 & 6.8 & 10.0 & 0.8 \\
\hline
\end{tabular}

\section{Results and discussion}

\subsection{Comparison of Mie theory and boundary element method}

Extinction spectra of single gold nanoparticles (18 and 40 $\mathrm{nm}$ in diameter) were calculated using Mie theory and boundary element method to compare the results of the two different approaches. For individual particles in water, the spectra calculated by the two different approaches show excellent agreement and agree with experimentally obtained data [12], with a slight difference in the peak intensity for the $18 \mathrm{~nm}$ gold nanoparticles (Figure 4). Hence, discretizing the nanoparticle surface using 400 vertices is sufficient for the accurate simulation of the optical response of these nanoparticle sizes. On the other hand, it enables time-efficient computation, although as the particle number increases, (especially for longer chains and 3D clusters) the simulation time also increases up to 12 hours.
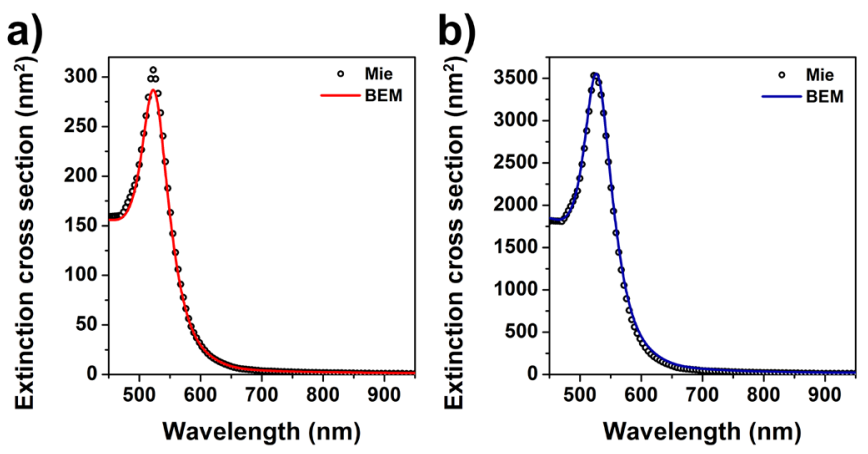

Fig. 4 Comparison of extinction cross sections calculated by Mie theory (open black circles) and the BEM simulation (solid lines) for single gold nanoparticles in diameter of $18 \mathrm{~nm}$ (a) and $40 \mathrm{~nm}$ (b) embedded in water.

\subsection{Optical simulations for linear chains}

Simulation data are presented for the longitudinal mode only, i.e. when light polarization is parallel to the chain axis. The reason to investigate only this mode (polarization direction) is that for coupled systems the strength of the coupled mode exceeds the single particle mode by orders of magnitude i.e. it becomes the optically dominant mode [19]. The structure itself is highly anisometric, hence besides the coupled (longitudinal) mode the transversal excitation contributes the overall optical response to a smaller extent as well, analogously to gold nanorods. The transversal mode is determined by the diameter of the particles and hence its position is independent on the number of particles in the cluster or chain, whereas the individual contribution of each particle adds up and contributes to the intensity of this mode. Figure 5 shows the simulated (longitudinal) extinction cross sections for linear chains built from $18 \mathrm{~nm}$ gold particles. The number of the building blocks (nanoparticles) was varied from 2 to 13 particles, and the interparticle separation distance was varied between $3.80 \mathrm{~nm}$ and $0.20 \mathrm{~nm}$.

For larger separation $(3.80 \mathrm{~nm})$ the particles are located relatively far from each other compared to the particle diameter (ca. $20 \%$ ), thus plasmon coupling does not influence the original spectrum of an individual particle dramatically. Still, a small broadening of the LSPR peak can be observed, indicating a minor overlap between the near fields of the neighboring particles already at this separation value. Decreasing the gap between the particles, a new resonance peak starts to evolve at higher wavelengths, indicating pronounced coupling for all chain lengths (number of particles in the chain). For small number of particles the initial LSPR peak remains pronounced as well showing a clear redshift, however starting with 4 NPs in the chain, its contribution to the overall extinction compared to the coupled mode becomes insignificant. As the separation 

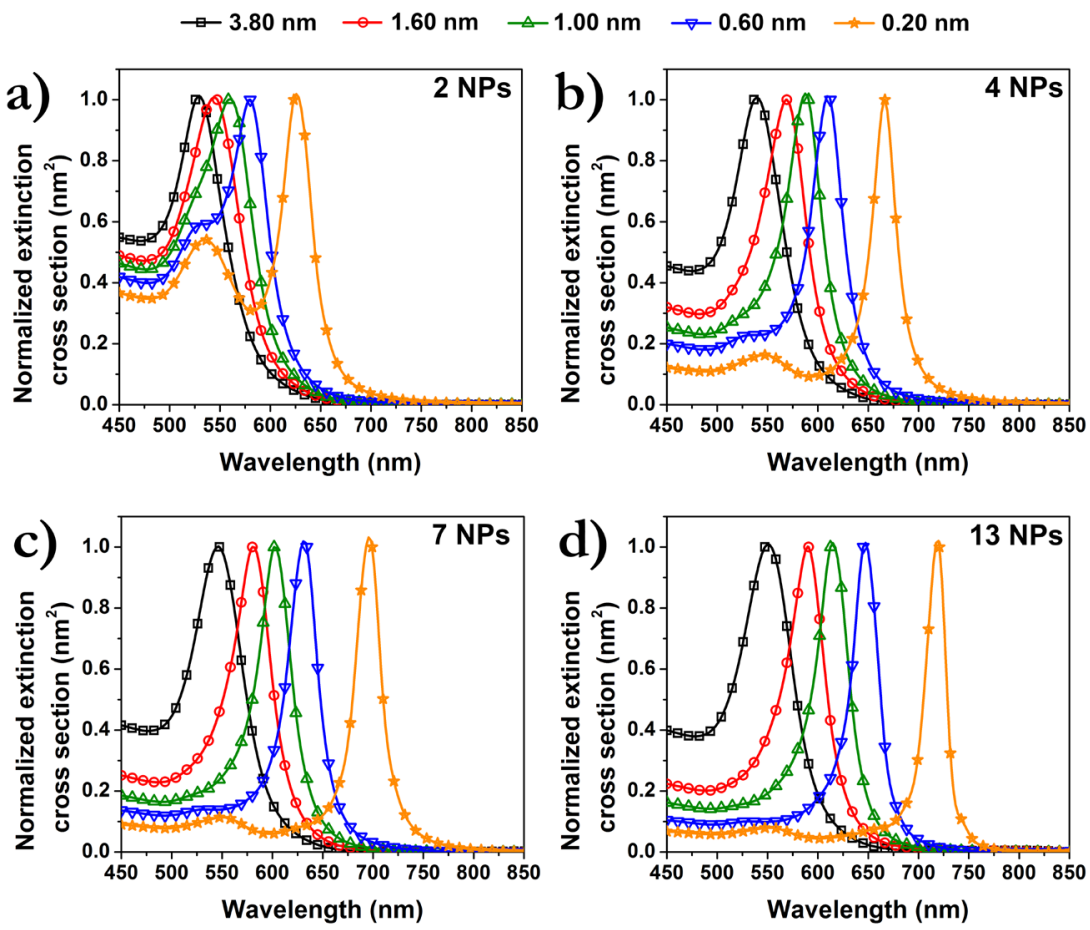

Fig. 5 Simulated normalized extinction spectra of $18 \mathrm{~nm}$ gold linear chains for different chain lengths and interparticle separation distances. Number of particles in the chains can be seen in the top right corners. Separation distance was varied from 0.2 to $3.8 \mathrm{~nm}$ which can be identified by the legend.
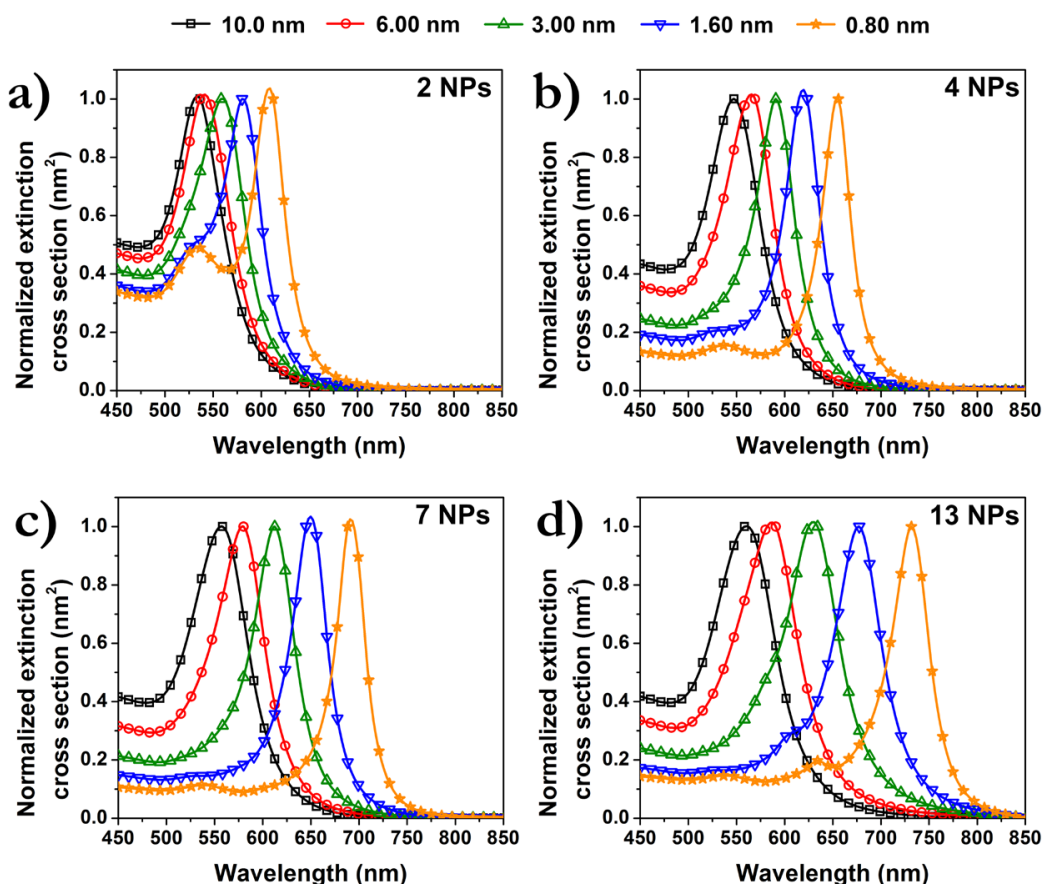

Fig. 6 Simulated normalized extinction spectra of $40 \mathrm{~nm}$ gold linear chains for different chain lengths and interparticle separation distances. Number of particles in the chains can be seen in the top right corners. Separation distance was varied from 0.8 to $10.0 \mathrm{~nm}$ which can be identified by the legend.

between the particles decreases, coupling manifests itself in the broadening of the LPSR band, which results in the appearance of a new coupled mode (around $0.60 \mathrm{~nm}$ separation distance).

Simulations were performed for chains of $40 \mathrm{~nm}$ gold nanoparticles as well and the results have been summarized in Fig. 6. The trends observed for the smaller particle size can be extended to this model system as well: if the particles are getting closer to each other, a redshift of the LSPR occurs and a new peak starts to evolve at higher wavelengths due to coupling.

These simulation results are in agreement with the ones obtained by Taylor et al. [19], who investigated the optical properties of quasi-fractal aggregates experimentally and theoretically as well. They compared time-resolved spectroscopic results with simulations for gold nanoparticles with diameters 
of 20 and $60 \mathrm{~nm}$; the aggregates featured a sub-nanometer $(0.9 \mathrm{~nm})$ interparticle gap. For $20 \mathrm{~nm}$ particles they found that coupled mode of dimers forms around $2.0 \mathrm{eV}(620 \mathrm{~nm})$ and chains can be found around $1.85 \mathrm{eV}(670 \mathrm{~nm})$.

Based on the experimental and simulation results it is obvious, that the gap size has a determining influence on extinction spectra. In a real aggregation experiment, surface attached molecules - acting as a spacer between the particles - provide this equilibrium separation. As observed in the simulated extinction spectra for chains, $1 \mathrm{~nm}$ interparticle distance causes only a moderate redshift to $600 \mathrm{~nm}$ for the $18 \mathrm{~nm}$ particles, even though a linear chain structure was assumed, which is characteristic for the diffusion limited aggregation as will be shown later in Fig. 8. Simulation results are in agreement with the observations of Lin et al. [30], who aggregated citrate stabilized $13 \mathrm{~nm}$ gold nanoparticles into branched chain network, and followed the process by time-dependent UV-Vis spectroscopy. Evolution of nanoparticle network manifested in a continual decrease in the $520 \mathrm{~nm}$ band with time and an increase and redshift of a shoulder from 630 to $705 \mathrm{~nm}$. Zhong et al. [31] simulated linear aggregates of gold nanoparticles $(20 \mathrm{~nm}$ and $40 \mathrm{~nm}$ in diameter) using DDA, as well. In this study, the number of particles in the chain and the separation distance was varied. Changing the gap between two $20 \mathrm{~nm}$ particles caused red-shift in LSPR band from $540 \mathrm{~nm}$ to $605 \mathrm{~nm}$ for $4.0 \mathrm{~nm}$ and $0 \mathrm{~nm}$ separations, respectively. Spectral shift was observed from 530 to $710 \mathrm{~nm}$, when the gap was set to $0.5 \mathrm{~nm}$ for $40 \mathrm{~nm}$ particles, and the number of nanoparticles in the chain was varied from 1 to 7. These previous results also support our observations on simulation of chain-like structures.

To highlight the coupled nature of the evolving new modes in our system, near field calculations have been carried out as well. Figure 7 shows the near fields of gold chain at separation distance of $1.60 \mathrm{~nm}$ at two different excitation wavelengths: at the wavelength of the single particle contribution $(537 \mathrm{~nm})$ and at the wavelength of the coupled mode $(645 \mathrm{~nm}$ ) (also see Fig. 6c for details). Near field plots indicate that the resonance peak at the longer wavelength is indeed related to coupling and a significant enhancement (well above 50) can be observed.

For the present study, we carried out the diffusion limited aggregation of $18 \mathrm{~nm}$ citrate stabilized gold nanoparticles to obtain the optical response of a complex, fractal-like aggregate structure. As shown in Fig. 8a, the aggregation results in a complex structure, which is essentially a branched nanoparticle network, where chains with different length and different branching ratio. The change of the extinction spectrum due to aggregation is shown in Fig. 8b: the initial spectrum of stable particles changes to a broad spectrum covering almost evenly the whole visible wavelength range above the LSPR of the starting nanoparticles.
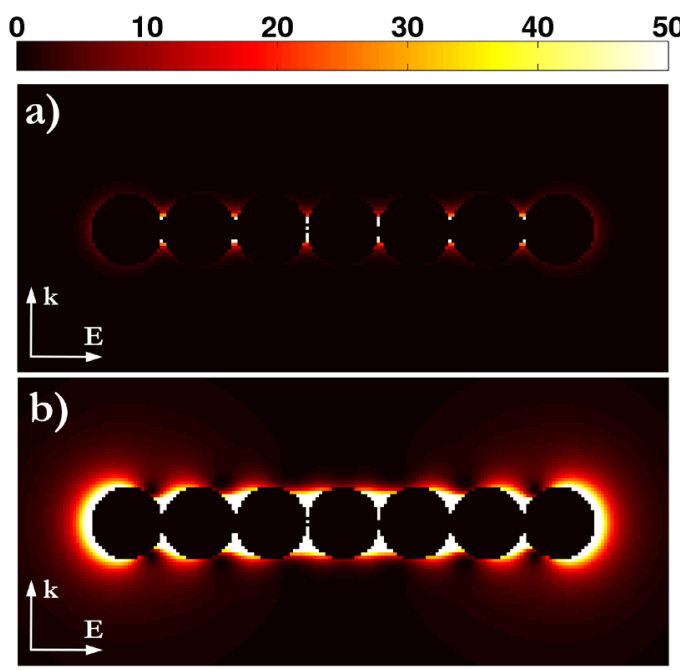

Fig. 7 Calculated near fields of gold chains of 7 nanoparticles (in diameter of $40 \mathrm{~nm}$ ) at separation distance of $1.60 \mathrm{~nm}$ : a) at $537 \mathrm{~nm}, \mathrm{~b}$ ) at $645 \mathrm{~nm}$.
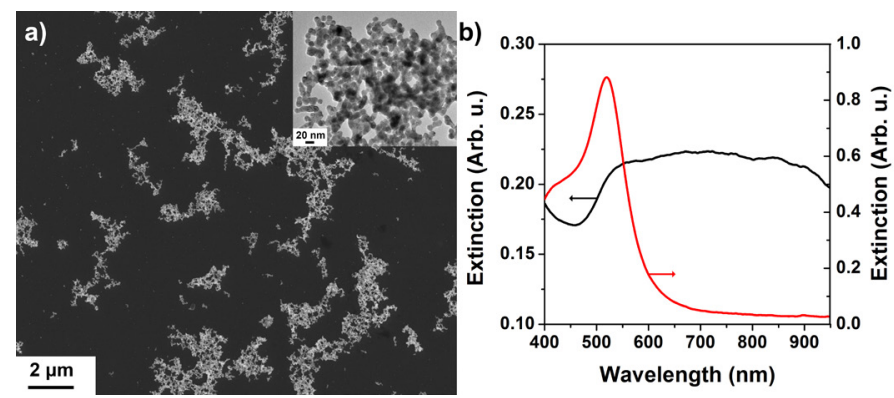

Fig. 8 Aggregation of citrate stabilized gold nanoparticles. SEM image of aggregates (a) and extinction spectra of citrate stabilized gold nanoparticles (b) measured before (red curve) and after (black curve) the increasing of ionic strength using $\mathrm{K}_{2} \mathrm{SO}_{4}$ solution. Inset of left panel shows the HR-TEM image of the aggregate.

This experimental result agrees with the simulated extinction spectra, where the optical response was found to depend sensitively on the chain length (number of particles) and together with the varying interparticle separation allows the coverage of the whole visible wavelength range. Based on the simulation result presented in Fig. 4 and 5, this also infers that such a full coverage of visible wavelength range (i.e. obtaining a greyish solution upon aggregation) can be achieved only if the particles are allowed to nearly touch each other in the aggregate thanks to the small sized ligand molecules on the gold surface. It also shows, that special care has to be taken when concluding solely from the extinction spectra on the structure of the prepared clusters, since even for the simplest case (1D linear chain) markedly different spectra can be obtained depending on the interparticle spacing. That is, when larger molecules are present on the surface, even in the aggregated state the spectra might show distinct extinction peaks, but this observation is not an evidence for the presence of three dimensional clusters, it might also originate from a linear, fractal-like assembly with 
larger interparticle spacing. For this reason, extinction measurement in itself does not provide evidence for the presence of special structures (3D structures or dimers), and complementary measurements (e.g. DLS or SEM) has to be performed.

\subsection{Optical simulations for 2D heptamers}

As a characteristic building block of self-assembled closed packed monolayer of particles, hcp structure built up from 7 nanoparticles was investigated for two different nanoparticle diameters. Figure 9 shows the results of simulation for extinction spectra at different interparticle separations. While the spectra did not change significantly from the individual particle's spectra at large separations $(3.8 \mathrm{~nm}$ for $18 \mathrm{~nm}$ particles and $10.0 \mathrm{~nm}$ for $40 \mathrm{~nm}$ particle, respectively), decreasing the gap causes redshift and broadening of the LSPR band. Due to the X-polarized light, the structure can be considered as a special type of chain, where the length of the chains is 3 particles. However, the presence of the other 4 particles with the chain results different extinction spectra. For this $2 \mathrm{D}$ array structure the observed coupled modes do not exceed $650 \mathrm{~nm}$, thus the optical response can be tuned only from $530 \mathrm{~nm}$ to $650 \mathrm{~nm}$, in contrast to the chain-like structures.
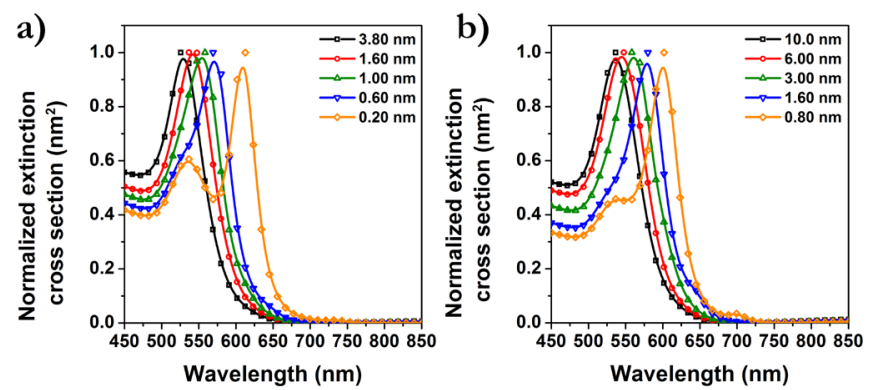

Fig. 9 Simulated extinction cross sections as a function of wavelength for 2D arrays at different separation distances for $18 \mathrm{~nm}$ (a) and $40 \mathrm{~nm}$ (b) gold nanoparticles.

Ben et al. carried out calculations using discrete dipole approximation for 2D infinite $f c c$ array of gold nanoparticles [32]. They investigated nanoparticles in a wide size range (20$100 \mathrm{~nm}$ ) and varied the gap between the particles and found that plasmon ruler equation is valid also for 2D arrays. Our results (presented in Fig. 9) are in excellent agreement with the LSPR shifts calculated by Ben and his co-workers for infinite arrays as shown in Figure 10a and b. Here, the peak shifts obtained from our simulation results for the different gap/particle diameter ratios as used in the calculations are plotted. For a convenient comparison, the values obtained from the plasmon ruler equation $[32,23]$ are also shown as a continuous line. a)

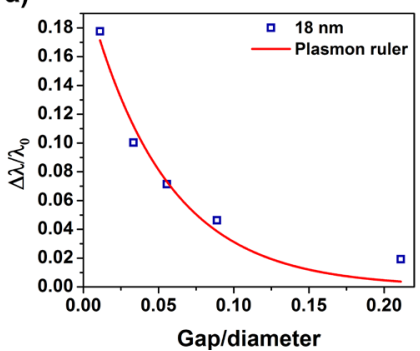

b)

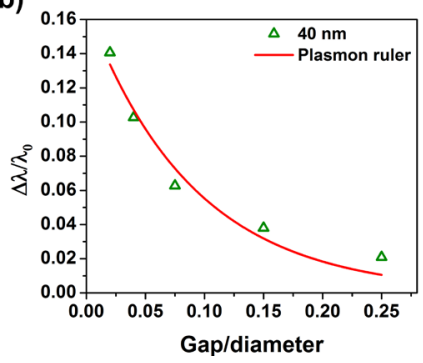

Fig. 10 LSPR peak shifts in a function of gap for $18 \mathrm{~nm}$ (a) and $40 \mathrm{~nm}$ (b) gold heptamers. Red curves illustrate the plasmon ruler function: $y=A \cdot \exp$ $\left[(\mathrm{x}-0.025) / \tau_{18 \mathrm{~nm}}\right]$ (a) and $\mathrm{y}=\mathrm{A} \cdot \exp -\left[(\mathrm{x}-0.025) / \tau_{40 \mathrm{~nm}}\right](\mathrm{b})$, where $\mathrm{y}$ is the $\Delta \lambda / \lambda_{0}$, $\mathrm{x}$ is the gap/diameter value, $\mathrm{A}$ is the preexponential constant $(0.131$ and 0.127 for 18 and $40 \mathrm{~nm}$, respectively) and $\tau$ is the decay parameter $\left(\tau_{18 \mathrm{~nm}}=0.053\right.$, $\left.\tau_{40 \mathrm{~nm}}=0.091\right)$.

The calculated near-field maps (Fig. 11) also indicate that the peak around $526 \mathrm{~nm}$ is related to the single particle contribution, but at $580 \mathrm{~nm}$ a coupled mode can be observed for the heptamer of $40 \mathrm{~nm}$ particles.

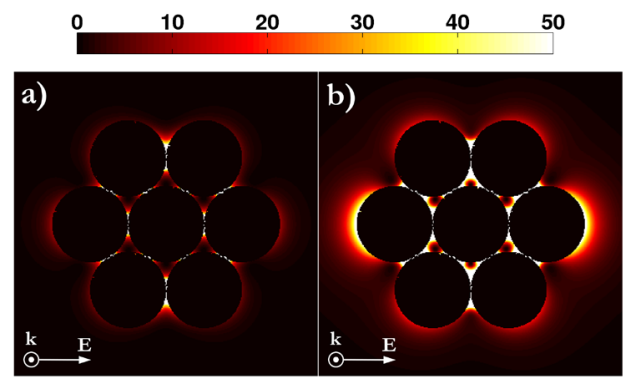

Fig. 11 Calculated near fields of gold heptamers of 7 nanoparticles (in diameter of $40 \mathrm{~nm}$ ) at separation distance of $1.60 \mathrm{~nm}$ : a) at $526 \mathrm{~nm}$, b) at 580 $\mathrm{nm}$. Color bar represents the near field enhancement.

\subsection{Optical simulations for 3D nanoparticle clusters}

Similarly to their 1D and 2D counterparts, three-dimensional nanoparticle clusters can have a complex optical response in the visible and near infrared region of wavelength, which is sensitive to the structural geometry [15]. For a better understanding of the optical properties of these ordered nanoclusters, it is important to know how the structure and the interparticle separation affects their spectra. A simple closed packed 3D structure built up from 13 nanoparticles was chosen for the optical simulations. Figure 12 demonstrates the obtained extinction spectra both for the 18 and $40 \mathrm{~nm}$ particles. For the smaller particles at the largest separation the cluster retains the optical properties of the individual nanoparticles, which shows that plasmon coupling in this case is not significant. Decreasing the gap to 0.6 $\mathrm{nm}$ or less causes continuous red-shift and broadening until 560 $\mathrm{nm}$, where a coupled mode evolves. Similar behavior can be observed for bigger nanoparticles (Fig. 12b), however, coupling can be already observed at separation distance of $3.0 \mathrm{~nm}$. 

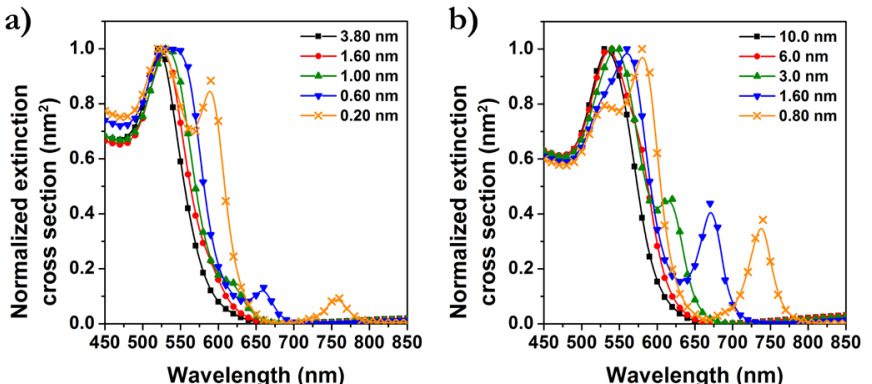

Fig. 12 Simulated extinction cross sections as a function of wavelength for $3 \mathrm{D}$ clusters at different separation distances for $18 \mathrm{~nm}$ (a) and $40 \mathrm{~nm}$ (b) gold nanoparticles.

For $40 \mathrm{~nm}$ particles, the near fields were calculated at two different wavelengths and three different planes. As shown in Fig. 13, the field enhancement for the $670 \mathrm{~nm}$ (bottom row) is significantly higher compared to $560 \mathrm{~nm}$ (top row). The small asymmetry in Figures a and d are due to retardation effects, whereas in $\mathrm{c}$ and $\mathrm{f}$ the high intensity region is located between the two particles aligned parallel to the excitation polarization.

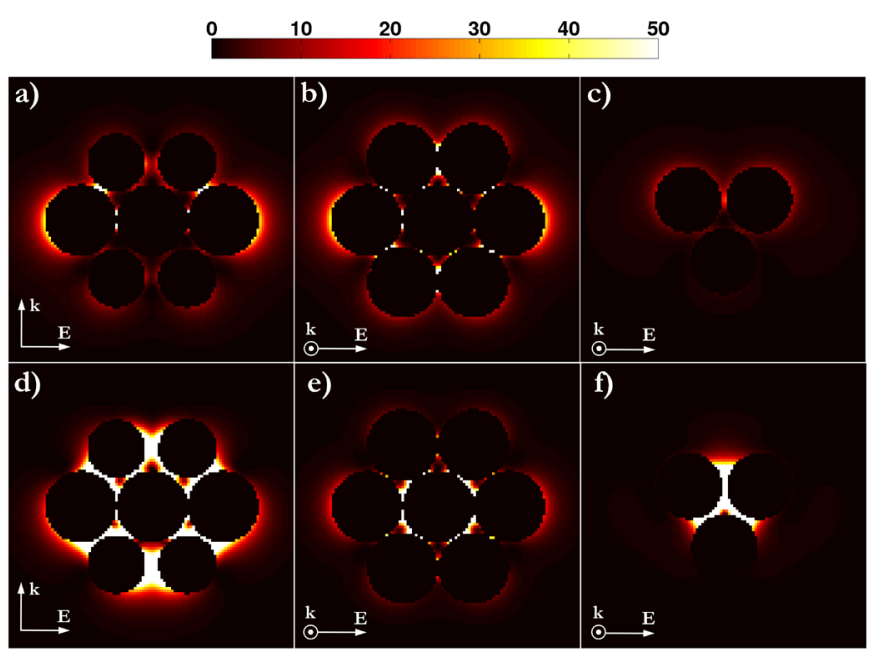

Fig. 13 Calculated near fields of 3D cluster of 13 nanoparticles (in diameter of $40 \mathrm{~nm}$ ) at separation distance of $1.60 \mathrm{~nm}$ for different slices: a-c) at 560 $\mathrm{nm}, \mathrm{d}-\mathrm{f}$ ) at $670 \mathrm{~nm}$. Direction (k) and polarization (E) of incident light is labeled in the bottom left corners of the images.

\section{Conclusion}

Simulated extinction spectra of linear chains, 2D heptamers and 3D clusters built up from gold nanoparticles have been calculated and compared. The simulations were carried out using boundary element method (BEM), where the particles were embedded into water and the separation distances were varied in order to map the optical response of relevant structures in colloidal self-assembly processes.

It is known, that diffusion-limited aggregation results in hyperbranched gold nanoparticle chains, thus extinction properties of chains were investigated for 2-13 numbers of particles. In accordance with experiments it was shown, that whole visible range can be covered by the extinction of the clusters, but only if surface-to-surface separations well below $1 \mathrm{~nm}$ apply.

Two-dimensional heptamers were investigated as the characteristic building block of infinite close packed layers, which are relevant in interfacial assemblies and nanoparticle monolayers. Our results are in good agreement with previously published results obtained for infinite arrays using DDA approach and conform well to the plasmon ruler equation.

The simulation results obtained for $3 \mathrm{D}$ nanocluster with internal order and made up of 13 nanoparticles show a lower intensity coupled mode compared to linear chains or 2D heptamers.

In general, for all types of particle assemblies (linear chains, $2 \mathrm{D}$ or $3 \mathrm{D}$ clusters), the determining factor in the evolution of the coupled mode was the interparticle gap, depending on which markedly different spectral shaping can be achieved even for the same type of cluster. This draws the attention to the importance on experimental design at the nanoparticle synthesis and surface modification level, since large surface molecules might limit the achievable coupling or spectral band in a certain application.

\section{Acknowledgement}

This work received funding from the European Union's Seventh Framework Programme for research, technological development and demonstration under the project UNION, Grant $N^{\circ} 310250$. The project was supported by the Hungarian Research Fund "OTKA-PD-105173" and "K-112114". A.D acknowledges the support of the János Bolyai Research Fellowship from the Hungarian Academy of Sciences. D.Z. acknowledges the support of the Pro Progressio Foundation and József Varga Foundation.

\section{References}

[1] Joseph, V., Matschulat, A., Polte, J., Rolf, S., Emmerling, F., Kneipp, J "SERS Enhancement of Gold Nanospheres of Defined Size." Journal of Raman Spectroscopy. 42(9), pp. 1736-1742. 2011.

DOI: $10.1002 /$ jrs.2939

[2] Saha, K., Agasti, S. S., Kim, C., Li, X., Rotello, V. M. "Gold Nanoparticles in Chemical and Biological Sensing." Chemical Society Reviews. 112(5), pp. 2739-2779. 2012. DOI: 10.1021/cr2001178

[3] Jans, H., Huo, Q. "Gold Nanoparticle-Enabled Biological and Chemical Detection and Analysis." Chemical Society Reviews. 41(7), pp. 2849 2866. 2012. DOI: 10.1039/C1CS15280G

[4] Panigrahi, S., Basu, S., Praharaj, S., Pande, S., Jana, S., Pal, A., Ghosh, S. K., Pal, T. "Synthesis and Size-Selective Catalysis by Supported Gold Nanoparticles: Study on Heterogeneous and Homogeneous Catalytic Process." The Journal of Physical Chemistry C. 111(12), pp. 4596-4605. 2007. DOI: $10.1021 /$ jp067554u

[5] Wu, H., Huang, X., Gao, M., Liao, X., Shi, B. "Polyphenol-Grafted Collagen Fiber as Reductant and Stabilizer for One-Step Synthesis of Size-Controlled Gold Nanoparticles and Their Catalytic Application to 4-Nitrophenol Reduction." Green Chemistry. 13(3), pp. 651-658. 2011. DOI: 10.1039/C0GC00843E 
[6] Dykman, L., Khlebtsov, N. "Gold Nanoparticles in Biomedical Applications: Recent Advances and Perspectives." Chemical Society Reviews. 41(6), pp. 2256-2282. 2012. DOI: 10.1039/C1CS15166E

[7] Yeh, Y.-C., Creran, B., Rotello, V. M. "Gold Nanoparticles: Preparation, Properties, and Applications in Bionanotechnology." Nanoscale. 4(6), pp. 1871-1880. 2012. DOI: 10.1039/C1NR11188D

[8] Njoki, P. N., Lim, I.-I. S., Mott, D., Park, H.-Y., Khan, B., Mishra, S., Sujakumar, R., Luo, J., Zhong, C.-J. "Size Correlation of Optical and Spectroscopic Properties for Gold Nanoparticles." The Journal of Physical Chemistry C. 111(40), pp. 14664-14669. 2007. DOI: $10.1021 / \mathrm{jp} 074902 \mathrm{z}$

[9] Eustis, S., El-Sayed, M. A. "Why Gold Nanoparticles Are More Precious than Pretty Gold: Noble Metal Surface Plasmon Resonance and Its Enhancement of the Radiative and Nonradiative Properties of Nanocrystals of Different Shapes." Chemical Society Reviews. 35(3), pp. 209-217. 2006. DOI: 10.1039/B514191E

[10] Gergely-Fülöp, E., Nagy, N., Deák, A. "Langmuir-Blodgett Films of Gold Nanorods with Different Silica Shell Thicknesses." Periodica Polytechnica Chemical Engineering. 59(2), pp. 104-110. 2015. DOI: 10.3311/PPch.7596

[11] Klinkova, A., Choueiri, R. M., Kumacheva, E. "Self-Assembled Plasmonic Nanostructures." Chemical Society Reviews. 43(11), pp. 3976-3991. 2014. DOI: 10.1039/C3CS60341E

[12] Zámbó, D., Radnóczi, G. Z., Deák, A. "Preparation of Compact Nanoparticle Clusters from Polyethylene Glycol-Coated Gold Nanoparticles by Fine-Tuning Colloidal Interactions." Langmuir. 31(9), pp. 2662-2668. 2015. DOI: 10.1021/la504600j

[13] Zohar, N., Chuntonov, L., Haran, G. "The Simplest Plasmonic Molecules: Metal Nanoparticle Dimers and Trimers." Journal of Photochemistry and Photobiology C: Photochemistry Reviews. 21, pp. 26-39. 2014. DOI: 10.1016/j.jphotochemrev.2014.10.002

[14] Liu, Y., Begin-Colin, S., Pichon, B. P., Leuvrey, C., Ihiawakrim, D., Rastei, M., Schmerber, G., Vomir, M., Bigot, J. Y. "Two Dimensional Dipolar Coupling in Monolayers of Silver and Gold Nanoparticles on a Dielectric Substrate." Nanoscale. 6(20), pp. 12080-12088. 2014. DOI: $10.1039 / C 4 N R 03292 \mathrm{~F}$

[15] Urban, A. S., Shen, X., Wang, Y., Large, N., Wang, H., Knight, M. W., Nordlander, P., Chen, H., Halas, N. J. "Three-Dimensional Plasmonic Nanoclusters." Nano Letters. 13(9), pp. 4399-4403. 2013. DOI: $10.1021 / \mathrm{n} 1402231 \mathrm{z}$

[16] Hu, B., Cao, X., Zhang, P. "Selective Colorimetric Detection of Glutathione Based on Quasi-Stable Gold Nanoparticles Assembly." New Journal of Chemistry. 37(12), pp. 3853-3856. 2013. DOI: 10.1039/c3nj00978e

[17] Zhang, L., Dai, L., Rong, Y., Liu, Z., Tong, D., Huang, Y., Chen, T. "Light-Triggered Reversible Self-Assembly of Gold Nanoparticle Oligomers for Tunable SERS." Langmuir. 31(3), pp. 1164-1171. 2015. DOI: $10.1021 / 1 \mathrm{a} 504365 \mathrm{~b}$

[18] Xi, C., Marina, P. F., Xia, H., Wang, D. "Directed Self-Assembly of Gold Nanoparticles into Plasmonic Chains." Soft Matter. 11(23), pp. 45624571. 2015. DOI: 10.1039/C5SM00900F

[19] Taylor, R. W., Esteban, R., Mahajan, S., Coulston, R., Scherman, O. A., Aizpurua, J., Baumberg, J. J. "Simple Composite Dipole Model for the Optical Modes of Strongly-Coupled Plasmonic Nanoparticle Aggregates." The Journal of Physical Chemistry C. 116(47), pp. 25044 25051. 2012. DOI: $10.1021 / \mathrm{jp} 308986 \mathrm{c}$
[20] Chen, C.-F., Tzeng, S.-D., Chen, H.-Y., Lin, K.-J., Gwo, S. "Tunable Plasmonic Response from Alkanethiolate-Stabilized Gold Nanoparticle Superlattices: Evidence of Near-Field Coupling." Journal of the American Chemical Society. 130(3), pp. 824-826. 2008. DOI: $10.1021 / \mathrm{ja} 0773610$

[21] Tao, A., Sinsermsuksakul, P., Yang, P. "Tunable Plasmonic Lattices of Silver Nanocrystals." Nature Nanotechnology. 2(7), pp. 435-440. 2007. DOI: 10.1038/nnano.2007.189

[22] Chen, T., Pourmand, M., Feizpour, A., Cushman, B., Reinhard, B. M. "Tailoring Plasmon Coupling in Self-Assembled One-Dimensional Au Nanoparticle Chains through Simultaneous Control of Size and Gap Separation." The Journal of Physical Chemistry Letters. 4(13), pp. 21472152. 2013. DOI: $10.1021 / \mathrm{jz} 401066 \mathrm{~g}$

[23] Sönnichsen, C., Reinhard, B. M., Liphardt, J., Alivisatos, A. P. A "Molecular Ruler Based on Plasmon Coupling of Single Gold and Silver Nanoparticles." Nature Biotechnology. 23(6), pp. 741-745. 2005. DOI: $10.1038 /$ nbt1100

[24] Inan, U. S., Marshall, R. A. "Numerical Electromagnetics: The FDTD Method." Cambridge University Press. 2011.

[25] Dhatt, G., Lefrançois, E., Touzot, G. "Finite Element Method." John Wiley \& Sons. 2012.

[26] Yang, W.-H., Schatz, G. C., Duyne, R. P. V. "Discrete Dipole Approximation for Calculating Extinction and Raman Intensities for Small Particles with Arbitrary Shapes." The Journal of Chemical Physics. 103(3), pp. 869-875. 1995. DOI: 10.1063/1.469787

[27] Rockstuhl, C., Salt, M. G., Herzig, H. P. "Application of the BoundaryElement Method to the Interaction of Light with Single and Coupled Metallic Nanoparticles." Journal of the Optical Society of America A. 20(10), pp. 1969-1973. 2003. DOI: 10.1364/JOSAA.20.001969

[28] Hohenester, U., Trügler, A. "MNPBEM-A Matlab Toolbox for the Simulation of Plasmonic Nanoparticles." Computer Physics Communications. 183(2), pp. 370-381. 2012. DOI: 10.1016/j.cpc.2011.09.009

[29] Johnson, P. B., Christy, R. W. "Optical Constants of the Noble Metals." Physical Review B. 6(12), pp. 4370-4379. 1972. DOI: 10.1103/PhysRevB.6.4370

[30] Lin, S., Li, M., Dujardin, E., Girard, C., Mann, S. "One-Dimensional Plasmon Coupling by Facile Self-Assembly of Gold Nanoparticles into Branched Chain Networks." Advanced Materials. 17(21), pp. 25532559. 2005. DOI: 10.1002/adma.200500828

[31] Zhong, Z., Patskovskyy, S., Bouvrette, P., Luong, J. H. T., Gedanken, A. "The Surface Chemistry of Au Colloids and Their Interactions with Functional Amino Acids." The Journal of Physical Chemistry B. 108(13), pp. 4046-4052. 2004. DOI: 10.1021/jp037056a

[32] Ben, X., Park, H. S. "Size Dependence of the Plasmon Ruler Equation for Two-Dimensional Metal Nanosphere Arrays." The Journal of Physical Chemistry C. 115(32), pp. 15915-15926. 2011. DOI: 10.1021/jp2055415 